\title{
A Method Of Automatic Identification On Power Oscillation
}

\author{
Fan Yang \\ School of Electrical and Electronic Engineering,North China Electric Power University (Baoding), \\ Baoding 071000, China \\ 2632460413@qq.com
}

Keywords: power oscillation, automatic identification, data smoothing, envelope detection, power system

\begin{abstract}
In order to identify the power oscillation of the power grid and take the corresponding measures quickly,a method of identification for power oscillation based on the measured signal curve in power system is proposed.The measured data is processed to extract the overall trend of the signal and combining with the technology of envelope detection and the soft threshold,the intelligent analysis of the power curve and the fast identification of the power oscillation is realized by the adaptive algorithm. The method can judge the beginning time of the disturbance within several cycles as well as overcome the shortcomings of the traditional method of hard threshold,which is not accurate enough.Finally,the effectiveness of the proposed method is verified by an example of power oscillation in the power grid.
\end{abstract}

\section{Introduction}

With the deepening of the marketization of the electric power industry,the scale of the interconnected power grid is expanding, and the characteristics of operation are increasingly complex,and so that the power system is becoming more and more extreme and the power oscillation has become a common technical problem in both the domestic and foreign power grid[1-5].However,the intelligent recognition is still difficult when the disturbance occurs.In the engineering practice,the method of threshold determination is mainly used.

The conventional method of power fluctuation is calculated as follows:

$$
\Delta P=\frac{P(t)-P(t-1)}{P(t-1)}
$$

The equation(1) shows the changeable degree of the power,and it represents a increase in the power when the deviation is positive,and shows a drop when negative.

Through setting corresponding threshold to different kinds of measured value, it is thought that the disturbance occurs in the system when the measured value exceeds the threshold value we have set. Although the aforementioned technical program has a high computing speed,it also has many obvious disadvantages such as the occurrence of error and the difficulty of setting threshold and so on.

Aiming at the problem of identification for power oscillation,based on the measured data,and in combination with historical experience,as well as using the technology of envelope detection to design the adaptive algorithm,the power oscillation can be automatically identified.

\section{Identification For Power Oscillation}

This method can be used for intelligent analysis and automatic identification for active power curve.The detection includes:the starting point of power oscillation,the end point of power oscillation and the trend of oscillation.

In general, there is always a fluctuation in the signal of active power,and the amplitude of the fluctuation is closely related to the realistic power system.Power oscillation means the phenomenon that the power signals in the sampling period of memory have a large range of fluctuations. The amplitude of the oscillation is much larger than that of the normal amplitude.And the correct detection of the characteristic of the oscillating signal is the key to determine the starting point of the 
oscillation.The oscillating identification of the active power of the power grid needs to be combined with the specific characteristics of the current power grid in order to determine the correct threshold for fluctuation of active power.According to the change of the active power signal in the unit time and the value of the threshold,the occurrence of the oscillation can be judged,and thus the starting point and end point of the oscillation could also be identified.During the oscillation, the dynamic change of the trend of the oscillation can be given by the algorithm of envelope detection.By adapting the power information after pretreatment,the features of the oscillation could be identified automatically.

The specific algorithm is as follows:

(1)Parameter initialization:the threshold per,the upper foot marker $U \_$ind and the lower foot marker D_ind;

(2)Enter the original data $D t$ in real time and fix the time interval $T$,extract each time an average value as the time period of an equivalent data and save it in the variable $A$;

(3)Smoothing the equivalent data $A$.Set the current data for $A(i)$ and forward shift amount for $D$, so the specific calculation formula is as follows, and the smoothed data is stored in the variable $B$;

$$
B(i)=\frac{\sum_{j=0}^{T-1} A(i-j)}{D}
$$

(4)Expand and fill with the data $B$,extend each data in $B$ to a data segment of length $T$,then get the data to be used below;

(5)Find the upper envelope:select the continuous d data from the starting point of the data,then find out the biggest data and its corresponding feet and save them in the two arrays named Up and U_ind correspondingly, and name the foot marker as index as well;

(6)Take index +1 as the starting point, repeat the step (3) and (4) until all data is completed,and then the upper envelope has been found;

(7)Find the lower envelope:select the continuous d data from the starting point of the data,then find out the smallest data and its corresponding feet and save them in the two arrays named Down and D_ind correspondingly, and name the foot marker as index as well;

(8)Take index +1 as the starting point, repeat the step (5) and (6) until all data is completed,and then the lower envelope has been found;

(9)Process Up and Down with linear interpolation, and the data after interpolation is named as U_A, D_A;

(10)Smoothing U_A,D_A and the original value,and mark the processed data as Temp_U,Temp_D,M;

(11)Identification of the oscillation:

$$
\begin{aligned}
& \text { if } \min (\text { Temp_U }-M, M-\text { Temp_U })>M * \text { per } \\
& \quad \text { oscillation occurs } \\
& \text { else } \\
& \quad \text { normal } \\
& \text { end }
\end{aligned}
$$

It should be noted that the value of the threshold percentage per will affect the results of the recognition in the specific implementation process. The value of per depends on the size of the index itself.Generally speaking, the smaller the value of per is, the more parts of the rise and fall will be found.Conversely, when the value of per is greater, the abnormal states will be found less. The value of per is suggest to be between 0.005 and 0.01 . 


\section{Case Analysis}

In this paper, according to the measured data of the active power grid in Shaaxin Province as an example, the proposed method is tested. The active power signal measured by WAMS is showed in figure 1.

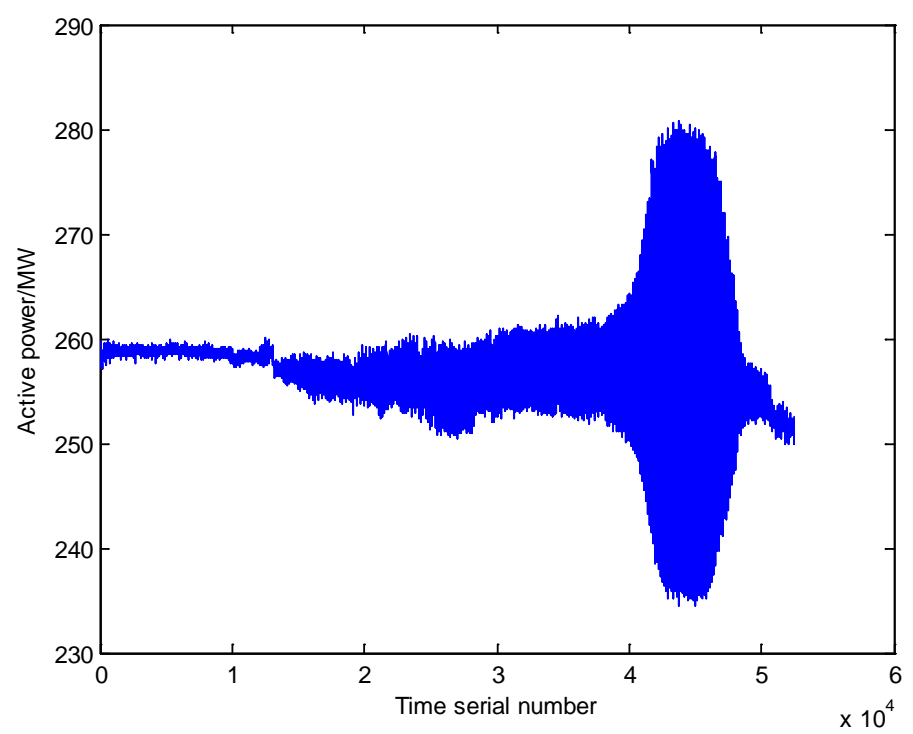

Fig 1 the measured data

And then Matlab programming is used to achieve the method to judge the oscillation of power signal in figure 1 .We set that per=0.006. And the effect of judgment is shown clearly in figure 2,in which the horizontal red line indicates the result of the original data smoothing,the green line indicates the upper envelope and the yellow line shows the lower envelope.It is evident that the method proposed in this paper can effectively judge the power oscillation.

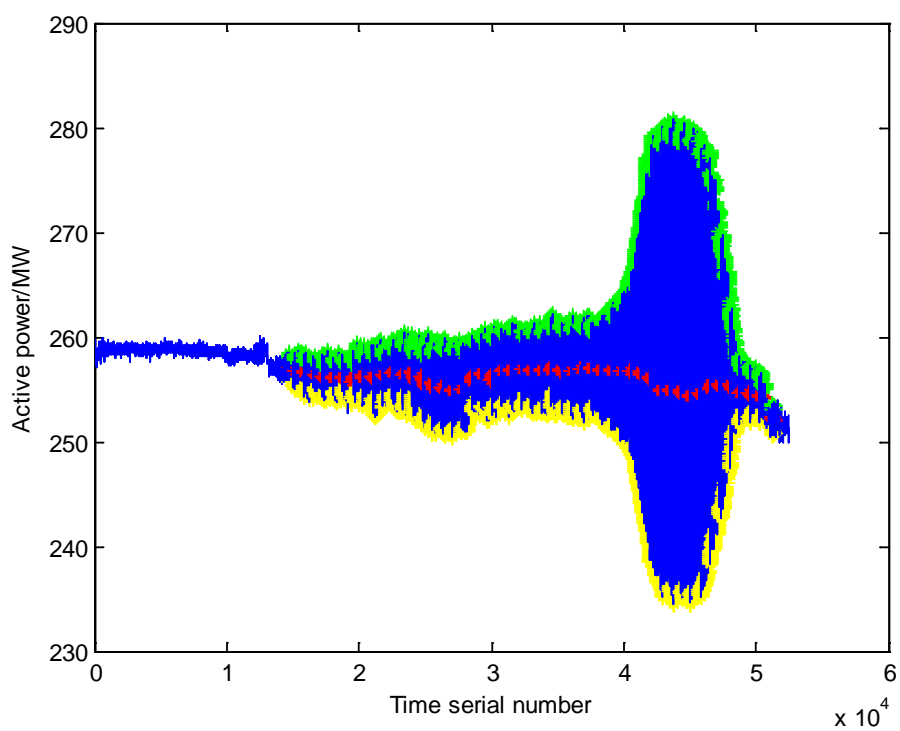

Fig 2 the effect of judgment

\section{Summary}

In order to identify the power oscillation of the power grid and take measures quickly,the method of identification for power oscillation based on the measured signal curve in power system is proposed.The method can effectively make use of the recording data of WAMS,PMU and other sets.Meanwhile,the practical method and the concrete steps of judging oscillation are presented from the point of view of engineering practicability in this paper.At the same time,the method overcomes 
the shortcomings of the traditional means to set hard threshold, which is easy to be disturbed by the noise and has difficulty in setting threshold.Since the experimental results has showed the effectiveness of the method,it is excellent enough to be used for on-line analysis to help improve the security and economy of grid operation.

\section{Acknowledgments}

This paper is funded by the University Students' Innovative Training Project.Finally,I would like to express my heartfelt thanks to Wang Tao and other teachers coming from Institute of mathematics and science,North China Electric Power University (Baoding)!

\section{References}

[1] Yong Huang,Lei Yun,Fei Tang,Work-energy transformation during forced power oscillation of power system,J. Electric Power Automation Equipment. 2013,33(8):41-46.

[2] Yiping Yu,Yong Min,Lei Chen,Analysis of Forced Power Oscillation Caused by Continuous Cyclical Load Disturbances, J. AUTOMATION OF ELECTRIC POWER SYSTEMS. 2010, 34(6):7-11,47.

[3] Dongjun Yang,Jianyong Ding,Hanqiao Shao,WAMS Based Characteristic Discrimination of Negative Damping Low-frequency Oscillation and Forced Power Oscillation,J.Automation of Electric Power Systems.2013,37(13):57-62.

[4] Yiping Yu,Yong Min,Lei Chen,Disturbance Source Location of Forced Power Oscillation Using Energy Functions,J.AUTOMATION OF ELECTRIC POWER SYSTEMS.2010,34(5):1-6.

[5] Ying Li,Wenshuang Jia, Wenfeng Li,Online Identification of Power Oscillation Properties Based on the Initial Period of Wave,J.Proceedings of the CSEE.2013,(25):54-60. 